\title{
Structural Properties of Optimal Coordinate-Convex Policies for CAC with Nonlinearly-Constrained Feasibility Regions
}

\author{
Marco Cello, Giorgio Gnecco, Mario Marchese and Marcello Sanguineti \\ Department of Communications, Computer and Systems Science (DIST) \\ University of Genoa - Via Opera Pia, 13 - 16145 Genova (Italy) \\ Email: marco.cello@dist.unige.it, giorgio.gnecco@dist.unige.it, mario.marchese@unige.it, marcello@dist.unige.it
}

\begin{abstract}
Necessary optimality conditions for Call Admission Control (CAC) problems with nonlinearly-constrained feasibility regions and two classes of users are derived. The policies are restricted to the class of coordinate-convex policies. Two kinds of structural properties of the optimal policies and their robustness with respect to changes of the feasibility region are investigated: 1) general properties not depending on the revenue ratio associated with the two classes of users and 2) more specific properties depending on such a ratio. The results allow one to narrow the search for the optimal policies to a suitable subset of the set of coordinate-convex policies.
\end{abstract}

Index Terms-Call Admission Control, Feasibility Region, Coordinate Convex Policies.

\section{INTRODUCTION}

Call Admission Control (CAC) represents an important mechanism to guarantee specific Quality of Service (QoS) requirements. CAC determines when to accept or reject a new connection, flow, or call request (depending on which technology is used), thus limiting the load that enters a network. This is accomplished by verifying if enough resources are available to satisfy the performance requirements of a new call without penalizing the ones already in progress.

A basic model for CAC is the stochastic knapsack [1]. In this model, one has $C$ units of resources and $K$ classes of users. The calls from each class $k \in\{1, \ldots, K\}$ arrive according to a Poisson process. If accepted by the system, each of them occupies $b_{k}$ units of resources, which are released at the end of the call. The simplest CAC policy, known as Complete Sharing $(C S)$, consists in accepting a call whenever the system has sufficient resources. However CS may lead to a monopolistic use of resources by certain classes of users, thus to a poor resource utilization [2, Section III]. This motivates the interest in different admission policies (see the references in [3, Section 7.1]).

In general, finding optimal policies for the stochastic knapsack model is a difficult combinatorial optimization problem [4, Chapter 4]. The knowledge of structural properties of the optimal policies is useful to simplify its solution or at least to find good suboptimal policies. For instance, for two classes of users and an objective given by a weighted sum of per-class average revenues, structural properties were derived in [1] for the optimal policies belonging to the class of coordinateconvex policies. Coordinate-convex policies form a large class of CAC policies characterized by a relatively simple structure and interesting properties, such as their product-form steadystate distribution [4, Chapter 4] and bounds on the per-class blocking probabilities [5]. When the service rates and resource requirements do not depend on the customer's class (single service), the optimal CAC policy is not coordinate-convex and is called trunk reservation ([6], [7]). For such a case, [8] and [9] provide recursive formulas to evaluate the performance of any trunk reservation policy and an iterative search algorithm to find optimal policies. They exploit such an algorithm to find coordinate optimal threshold policies (a particular kind of coordinate-convex policies) in multiservice systems (for which different classes may have different and heterogeneous resource requirements and mean service times).

The stochastic knapsack model can be extended by introducing the concept of feasibility region [10, pp. 46-49]. This is a region $\Omega_{F R}$ in the call space where given QoS requirements in terms of packet-loss/packet-delay probability are statistically guaranteed. When some form of statistical multiplexing is used, often this implies that the linear constraint $\left\{\sum_{k \in K} n_{k} b_{k} \leq C\right\}$ for the stochastic knapsack model is replaced by a more complicated constraint for the feasibility region, e.g. of the form $\sum_{k \in K} \beta_{k}\left(n_{k}\right) \leq C$ [4, p. 212], where the $\beta_{k}(\cdot)$ are nonlinear functions (see Figure 1).

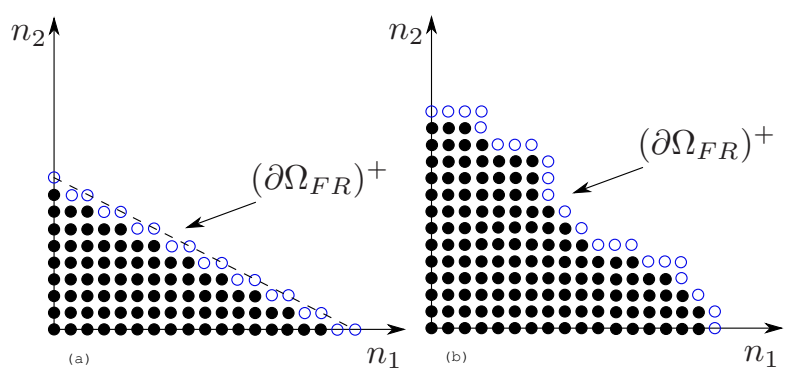

Figure 1. The upper boundary $\left(\partial \Omega_{F R}\right)^{+}$of a feasibility region $\Omega_{F R}$ with 2 class or users in the case of (a) a linearly-constrained $\Omega_{F R}$ (stochastic knapsack) and (b) a nonlinearly-constrained $\Omega_{F R}$.

Up to our knowledge, till now the problem of finding structural properties of the optimal coordinate-convex policies in the case of general nonlinearly-constrained feasibility regions has received little attention, with the exception of [11], [12] 
(see Subsection III-B for a comparison of our results with those of [12]).

The following is a summary of our contributions. For CAC problems with nonlinearly-constrained feasibility regions $\Omega_{F R}$ and two classes of users, we provide

- some general structural properties holding for any optimal coordinate-convex policy (Subsection III-A);

- more specific structural properties dependent on the revenue ratio associated with the two classes of users;

- simulation results (Section IV).

In doing so, we extend some results of [1] to nonlinearlyconstrained feasibility regions; see Subsections III-A and III-B. All the proofs are deferred to Section VI.

\section{PROBLEM FORMULATION}

In this section, we summarize the CAC problem studied in [1], which will be extended in Section III to a nonlinearlyconstrained feasibility region $\Omega_{F R}$.

The state of the CAC system in [1] is described by a 2-dimensional vector $\mathbf{n}$, whose component $n_{k}, k=1,2$, represents the number of connections from users of class $k$ that have been accepted by the system and are currently in progress. For each class $k$, the inter-arrival times are exponentially distributed with mean value $1 / \lambda_{k}\left(n_{k}\right)$ and the holding times of accepted connections are independent and identically distributed (i.i.d.) with mean value $1 / \mu_{k}$. The CAC system accepts or rejects a request of connection according to a coordinate-convex policy. Here we recall its definition [4, $\mathrm{p}$. 116].

Definition II.1. A nonempty set $\Omega \subseteq \Omega_{F R} \subset \mathbb{N}_{0}^{2}$ is called coordinate-convex if and only if it has the following property: for each $\mathbf{n} \in \Omega$ with $n_{k}>0$ one has $\mathbf{n}-\mathbf{e}_{k} \in \Omega$, where $\mathbf{e}_{k}$ is a 2-dimensional vector whose $k$-th component is 1 and the other one is 0 . The coordinate-convex policy associated with a coordinate-convex set $\Omega$ admits an arriving request of connection if and only if after admittance the state process remains in $\Omega$.

As there is a one-to-one correspondence between coordinateconvex sets and coordinate-convex policies, from now on we use the symbol $\Omega$ to denote either a coordinate-convex set or a coordinate-convex policy.

The objective to be maximized by the CAC system in the space $\mathcal{P}\left(\Omega_{F R}\right)$ of coordinate-convex subsets of $\Omega_{F R}$ is given by

$$
J(\Omega)=\sum_{\boldsymbol{n} \in \Omega}(\boldsymbol{n} \cdot \boldsymbol{r}) P_{\Omega}(\boldsymbol{n}),
$$

where $\mathbf{r}$ is a 2-dimensional vector whose component $r_{k}$ represents the instantaneous positive revenue generated by any accepted connection of class $k$ that is still in progress and $P_{\Omega}(\boldsymbol{n})$ is the steady-state probability that the CAC system is in state $\mathbf{n}$. As $\Omega$ is coordinate-convex, $P_{\Omega}(\boldsymbol{n})$ takes on the product-form expression

$$
P_{\Omega}(\boldsymbol{n})=\frac{\prod_{i=1}^{2} q_{i}\left(n_{i}\right)}{\sum_{\boldsymbol{n} \in \Omega} \prod_{i=1}^{2} q_{i}\left(n_{i}\right)},
$$

where

$$
q_{i}\left(n_{i}\right):=\frac{\prod_{j=0}^{n_{i}-1} \lambda_{i}(j)}{n_{i} ! \mu_{i}^{n_{i}}} .
$$

For linearly-constrained feasibility regions $\Omega_{F R}$, [1] describes structural properties of the coordinate-convex policies maximizing the objective (1) (e.g., the existence of one vertical threshold, one horizontal threshold, or both kinds of thresholds), which depend on the value assumed by the revenue ratio $R:=r_{2} / r_{1}$.

\section{MAIN RESULTS}

In our analysis, we allow the feasibility region $\Omega_{F R}$ to have a nonlinear upper boundary, denoted by $\left(\partial \Omega_{F R}\right)^{+}$(see Figure 1(b)). Similarly, we denote by $(\partial \Omega)^{+}$the (linear or nonlinear) upper boundary of the coordinate-convex set $\Omega$. The set $\Omega_{F R}$ is assumed to be coordinate-convex, as it often happens for feasibility regions defined in terms of QoS constraints [13, Proposition 6.3]. Let us recall two definitions from [1].

Definition III.1. The tuple $(\alpha, \beta) \in \Omega_{F R} \backslash \Omega$ is a type-1 corner point for $\Omega$ if and only if $\beta \geq 1,(\alpha, \beta-1) \in \Omega$, and either $\alpha=0$ or $(\alpha-1, \beta) \in \Omega$; the tuple $(\alpha, \beta) \in \Omega_{F R} \backslash \Omega$ is a type- 2 corner point for $\Omega$ if and only if $\alpha \geq 1,(\alpha-1, \beta) \in \Omega$, and either $\beta=0$ or $(\alpha, \beta-1) \in \Omega$.

Definition III.2. A nonempty set $S^{-} \subset \Omega_{F R}$ is incrementally removable with respect to $\Omega\left(I R_{\Omega}\right)$ if and only if $S^{-} \subset \Omega$ and $\Omega \backslash S^{-}$is still a coordinate-convex set; a nonempty set $S^{+} \subset \Omega_{F R}$ is incrementally admissible with respect to $\Omega$ $\left(I A_{\Omega}\right)$ if and only if $S^{+} \cap \Omega=\emptyset$ and $\Omega \cup S^{+}$is still a coordinateconvex set.

In the following, we shall sometimes use the term "corner point" to refer to either a type- 1 or a type- 2 corner point. By the coordinate-convexity of $\Omega$, no two corner points can be on the same vertical or horizontal line.

We recall from [1] that the definition of the objective $J(\cdot)$ in (1) can be extended consistently to all (not necessarily coordinate-convex) sets $S \subseteq \Omega_{F R}$ in the following way:

$$
J(S):=\frac{H(S)}{G(S)},
$$

with

$$
\begin{array}{r}
H(S):=\sum_{\boldsymbol{n} \in S}(\boldsymbol{n} \cdot \boldsymbol{r}) \prod_{i=1}^{2} q_{i}\left(n_{i}\right) \\
G(S):=\sum_{\boldsymbol{n} \in S} \prod_{i=1}^{2} q_{i}\left(n_{i}\right) .
\end{array}
$$

We also recall that, for a rectangular region $S:=\{a, a+$ $1, \ldots, b\} \times\{c, c+1, \ldots, d\}$, by (3), (5), and (6) it follows

$$
J(S)=r_{1} x_{1}(a, b)+r_{2} x_{2}(c, d),
$$

where

$$
x_{i}(a, b):=\frac{\sum_{k=a}^{b} k q_{i}(k)}{\sum_{j=a}^{b} q_{i}(j)} .
$$


A. General structural properties of the optimal coordinateconvex policies

Let $\Omega^{o}$ denote any optimal coordinate-convex policy (or its associated coordinate-convex set). Proposition III.3, which extends to nonlinearly-constrained feasibility regions a similar property stated in [1, Theorem 1] for linearly-constrained ones, states that the corner points of $\Omega^{\circ}$ are to be searched among the vertices of a suitable grid (see Figure 2). We use the following notations:

$$
\begin{aligned}
& l_{2}^{\Omega}\left(n_{1}\right):=\max \left\{k \in \mathbb{N}_{0} \text { such that }\left(n_{1}, k\right) \in \Omega\right\}, \\
& l_{1}^{\Omega}\left(n_{2}\right):=\max \left\{h \in \mathbb{N}_{0} \text { such that }\left(h, n_{2}\right) \in \Omega\right\} .
\end{aligned}
$$

The values $l_{1}^{\Omega}\left(n_{2}\right)$ and $l_{2}^{\Omega}\left(n_{1}\right)$ are, respectively, the maximum number of type-1/type- 2 connections allowed in $\Omega$ when we have already $n_{2}$ type- $2 / n_{1}$ type- 1 connections. It follows from the definitions that the functions $l_{i}^{\Omega}(\cdot)$ are nonincreasing. Set $n_{1, \max }:=l_{1}^{\Omega_{F R}}(0), n_{2, \max }:=l_{2}^{\Omega_{F R}}(0), l_{1}^{\Omega_{F R}}\left(n_{2, \max }+1\right)+$ $1:=0$ and $l_{2}^{\Omega_{F R}}\left(n_{1, \max }+1\right)+1:=0$.

Proposition III.3. (i) If $(\alpha, \beta)$ is a type-2 corner point for $\Omega^{o}$, then for some $j=1, \ldots, n_{2, \max }$ we have

$$
\alpha=l_{1}^{\Omega_{F R}}(j)+1 .
$$

(ii) If $(\alpha, \beta)$ is a type-1 corner point for $\Omega^{\circ}$, then for some $j=1, \ldots, n_{1, \max }$ we get

$$
\beta=l_{2}^{\Omega_{F R}}(j)+1 .
$$

We refer to the reader to [14] for other general structural properties of the optimal coordinate-convex policies, not depending on the revenue ratio $R$.

\section{B. Structural properties of the optimal coordinate-convex poli-} cies depending on the revenue ratio $R$

Let us now consider for the optimal coordinate-convex policies structural properties obtained for suitable values of the revenue ratio $R$. We recall the following definition from [1].

Definition III.4. Let $i=1$ or 2 . A coordinate-convex policy $\Omega$ is threshold type- $i$ if and only if for some $t_{i}=0, \ldots, n_{i, \max }$ we get

$$
\Omega=\left\{\left(n_{1}, n_{2}\right) \in \Omega_{F R}: n_{i} \leq t_{i}\right\} .
$$

Proposition III.5 is our extension of [1, Lemma 4] to general nonlinearly-constrained feasibility regions. With respect to [1], due to the different shape of the feasibility region, in general it is not true that $j \neq k$ implies $l_{1}^{\Omega_{F R}}(j) \neq l_{1}^{\Omega_{F R}}(k)$. As shown in Figure 2 , in general for every $j_{2} \in\left\{0, \ldots, n_{2, \max }\right\}$ there exist $j^{(2, l)} \leq j_{2}$ and $j^{(2, u)} \geq j_{2}$ such that $l_{1}^{\Omega_{F R}}(\cdot)$ is constant on the set $\left\{j^{(2, l)}, \ldots, j^{(2, u)}\right\} \subseteq\left\{0, \ldots, n_{2, \max }\right\}$. Similarly, for every $j_{1} \in\left\{0, \ldots, n_{1, \max }\right\}$ there exist $j^{(1, l)} \leq j_{1}$ and $j^{(1, u)} \geq j_{1}$ such that $l_{2}^{\Omega_{F R}}(\cdot)$ is constant on the set $\left\{j^{(1, l)}, \ldots, j^{(1, u)}\right\} \subseteq$ $\left\{0, \ldots, n_{1, \max }\right\}$. Let $B_{1}:=\max \left\{j^{(1, u)}-j^{(1, l)}: j_{1}=\right.$ $\left.0, \ldots, n_{1, \max }\right\}$ and $B_{2}:=\max \left\{j^{(2, u)}-j^{(2, l)}: j_{2}=\right.$ $\left.0, \ldots, n_{2, \max }\right\}$. Recall that $R:=r_{2} / r_{1}$ is the revenue ratio.

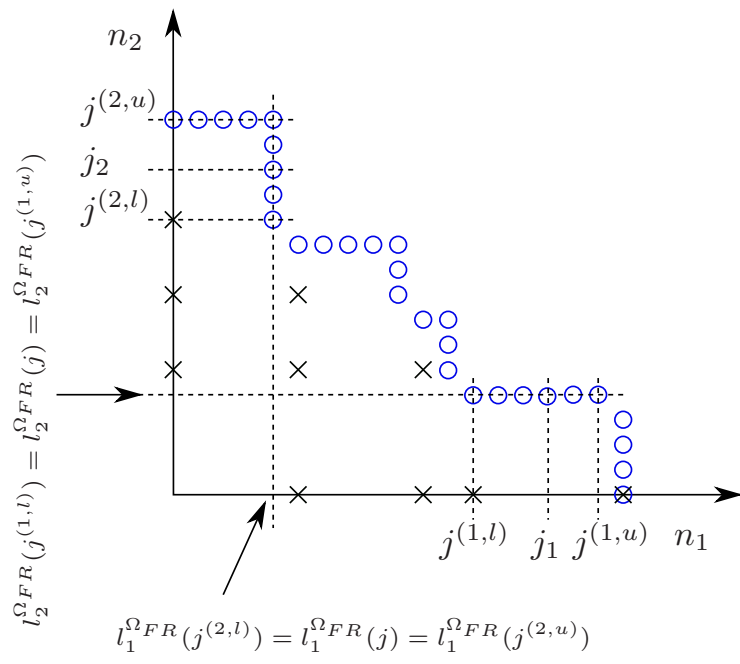

Figure 2. Potential locations of the corner points of an optimal coordinateconvex policy $\Omega^{\circ}$. According to Proposition III.3, the corner points of $\Omega^{\circ}$ are to be searched among the crosses in the figure.

Proposition III.5. (i) If $(\alpha, \beta)$ is a type-2 corner point for $\Omega^{o}$ and $\lambda_{2}(\cdot)$ is nonincreasing, then for some $j=1, \ldots, n_{2, \max }$ (11) holds together with

$$
\begin{array}{r}
R x_{2}\left(0, B_{2}\right) \geq x_{1}\left(l_{1}^{\Omega_{F R}}\left(j^{(2, l)}\right)+1, l_{1}^{\Omega_{F R}}\left(j^{(2, l)}-1\right)\right) \\
-x_{1}\left(l_{1}^{\Omega_{F R}}\left(j^{(2, u)}+1\right)+1, l_{1}^{\Omega_{F R}}\left(j^{(2, u)}\right)\right) .
\end{array}
$$

(ii) If $(\alpha, \beta)$ is a type-1 corner point for $\Omega^{o}$ and $\lambda_{1}(\cdot)$ is nonincreasing, then for some $j=1, \ldots, n_{1, \max }$ (12) holds together with

$$
\begin{aligned}
\frac{1}{R} x_{1}\left(0, B_{1}\right) & \geq x_{2}\left(l_{2}^{\Omega_{F R}}\left(j^{(1, l)}\right)+1, l_{2}^{\Omega_{F R}}\left(j^{(1, l)}-1\right)\right) \\
& -x_{2}\left(l_{2}^{\Omega_{F R}}\left(j^{(1, u)}+1\right)+1, l_{2}^{\Omega_{F R}}\left(j^{(1, u)}\right)\right) .
\end{aligned}
$$

The following theorem states that under suitable conditions one has threshold-type optimal policies. The result is an extension of [1, Theorem 1] to feasibility regions with a nonlinear upper boundary. Its proof exploits Proposition III.5.

Theorem III.6. Let $\lambda_{i}(\cdot)$ be nonincreasing for $i=1,2$.

(i) If $\frac{1}{R}<L_{1}$, where

$$
\begin{aligned}
L_{1}: & =\min _{j=1, \ldots, n_{1, \max }} \\
& \left\{\frac{x_{2}\left(l_{2}^{\Omega_{F R}}\left(j^{(1, l)}\right)+1, l_{2}^{\Omega_{F R}}\left(j^{(1, l)}-1\right)\right)}{x_{1}\left(0, B_{1}\right)}\right. \\
& \left.-\frac{x_{2}\left(l_{2}^{\Omega_{F R}}\left(j^{(1, u)}+1\right)+1, l_{2}^{\Omega_{F R}}\left(j^{(1, u)}\right)\right)}{x_{1}\left(0, B_{1}\right)}\right\},
\end{aligned}
$$

then $\Omega^{o}$ is threshold type-1, and the threshold is equal to some $l_{1}^{\Omega_{F R}}(j)$ for some $j=0, \ldots, n_{2, \max }$. 
(ii) If $R<L_{2}$, where

$$
\begin{aligned}
L_{2}:= & \min _{j=1, \ldots, n_{2, \max }} \\
& \left\{\frac{x_{1}\left(l_{1}^{\Omega_{F R}}\left(j^{(2, l)}\right)+1, l_{1}^{\Omega_{F R}}\left(j^{(2, l)}-1\right)\right)}{x_{2}\left(0, B_{2}\right)}\right. \\
& \left.-\frac{x_{1}\left(l_{1}^{\Omega_{F R}}\left(j^{(2, u)}+1\right)+1, l_{1}^{\Omega_{F R}}\left(j^{(2, u)}\right)\right)}{x_{2}\left(0, B_{2}\right)}\right\},
\end{aligned}
$$

then $\Omega^{\circ}$ is threshold type-2, and the threshold is equal to some $l_{2}^{\Omega_{F R}}(j)$ for some $j=0, \ldots, n_{1, \max }$.

(iii) If $\frac{1}{L_{1}}<R<L_{2}$, then $\Omega^{o}=\Omega_{F R}$.

The following corollary provides sufficient conditions for threshold-type optimal policies.

Corollary III.7. Let $\lambda_{i}(\cdot)$ be nonincreasing for $i=1,2$.

(i) If $R>x_{1}\left(0, B_{1}\right)$, then $\Omega^{o}$ is threshold type-1, and the threshold is equal to some $l_{1}^{\Omega_{F R}}(j)$ for some $j=0, \ldots, n_{2, \max }$.

(ii) If $\frac{1}{R}>x_{2}\left(0, B_{2}\right)$, then $\Omega^{o}$ is threshold type-2, and the threshold is equal to some $l_{2}^{\Omega_{F R}}(j)$ for some $j=0, \ldots, n_{1, \max }$.

(iii) If $x_{1}\left(0, B_{1}\right)<R<\frac{1}{x_{2}\left(0, B_{2}\right)}$, then $\Omega^{o}=\Omega_{F R}$.

Remark III.8. In the particular case of a linearly-constrained feasibility region with $B_{2}=1$ (i.e., the one considered in $[1])$, one has $j^{(1, l)}=j^{(1, u)}$ for each $j=0, \ldots, n_{1, \max }$, and $L_{1}=\frac{1}{x_{1}\left(0, B_{1}\right)}$. So in this case Theorem III.6 (i) reduces to [1, Theorem 1] (i).

Remark III.9. Another extension of [1, Theorem 1] which is similar to Proposition III.3 and Corollary III.7 is reported in [11, Section 4] and refers to a less general nonlinearlyconstrained feasibility region and to a different assumption on the holding time distribution of the calls.

\section{Simulation RESULTS}

In the next numerical results we show that, under the conditions of Corollary III.7 (i) and (ii) resp., the optimal threshold for threshold type- 1 policies is indeed equal to $l_{1}^{\Omega_{F R}}(j)$ for some $j=0, \ldots, n_{2, \max }$, and the optimal threshold for threshold type-2 policies is equal to $l_{2}^{\Omega_{F R}}(j)$ for some $j=0, \ldots, n_{1, \max }$. In Figure 3 the feasibility region used to make these simulations is depicted. We assume homogeneous Poisson arrivals for both classes.

With this feasibility region we have $B_{1}=16$ and $B_{2}=10$; for $\lambda_{1}=50, \lambda_{2}=150, \mu_{1}=0.5, \mu_{2}=5, r_{1}=0.25$, and $r_{2}=2.5$ we have $R=r_{2} / r_{1}=10$ and $x_{1}(0,10) \simeq 9.89$. Then $R>x_{1}\left(0, B_{1}\right)$ and by Corollary III.7 (i) there exists an optimal coordinate-convex policy that is threshold type- 1 . According to Corollary III.7 (i), the optimal threshold belongs to the set $\{0,15,22,28\}$. Figure 3 (b) shows that this is indeed the case, and that the optimal threshold is $t_{1}=15$.

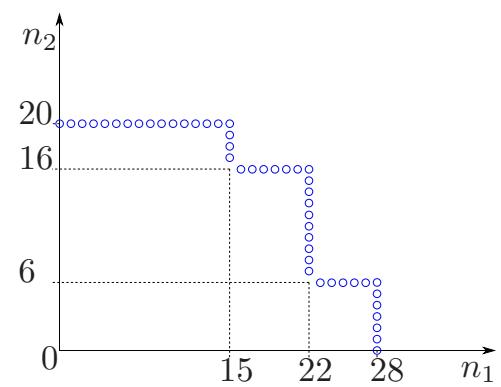

(a)

\begin{tabular}{|c|c|}
\hline Threshold $t_{1}$ & $J(\Omega)$ \\
\hline \hline 15 & 50.2007 \\
14 & 49.9540 \\
13 & 49.7072 \\
12 & 49.4604 \\
11 & 49.2135 \\
10 & 48.9665 \\
16 & 47.0714 \\
17 & 43.5764 \\
20 & 42.6669 \\
18 & 42.4987 \\
19 & 42.4718 \\
\hline
\end{tabular}

(b)
Figure 3. (a) The feasibility region considered in Section IV. (b)

\section{CONCLusions}

This paper provides several structural properties of the corner points of the optimal coordinate-convex policies in CAC problems with nonlinearly-constrained feasibility regions and two classes of users. These properties can be used to narrow the search for the optimal coordinate-convex policies. For certain feasibility regions characterized by a nonlinear constraint of the form $\sum_{k \in K} \beta_{k}\left(n_{k}\right) \leq C$, the simplest possible extension of the results to more than 2 classes of users consists in defining subproblems obtained by partitioning the set of classes by using subsets of cardinality at most 2 and applying to each subproblem the results obtained here for 2 classes of users.

\section{Proofs}

Due to the page limits, we refer the reader to [1] for the statements of [1, Lemmas 1-3], which are used in some of the following proofs. An inspection of the proofs of [1, Lemmas 1-3] shows that they hold for both linearly-constrained and nonlinearly-constrained feasibility regions.

Proof of Proposition III.3. We prove only (i), since (ii) can be obtained in the same way by exchanging the roles of the two classes of users. Suppose that (11) is violated for every $j=$ $1, \ldots, n_{2, \max }$. Choosing $n=l_{2}^{\Omega^{o}}(\alpha-1)-\beta \geq 0, S^{-}(n)=$ $\{(\alpha-1, \beta+i): i=0, \ldots, n\} \subseteq \Omega^{o}$, and $S^{+}(n)=\{(\alpha, \beta+i)$ : $i=0, \ldots, n\} \subseteq \Omega_{F R} \backslash \Omega^{o}$ (see Figure 4), it follows that the sets $\Omega^{o} \backslash S^{-}(n)$ and $\Omega^{o} \cup S^{+}(n)$ are coordinate-convex, so $S^{-}(n)$ is $I R_{\Omega^{\circ}}$ and $S^{+}(n)$ is $I A_{\Omega^{\circ}}$. By formula (7), we get $J\left(S^{-}(n)\right)=r_{1}(\alpha-1)+r_{2} x_{2}(\beta, \beta+n)<r_{1} \alpha+r_{2} x_{2}(\beta, \beta+$ $n)=J\left(S^{+}(n)\right)$, but this contradicts the optimality condition stated in [1, Lemma 2], so one concludes that there exists some $j=1, \ldots, n_{2, \max }$ such that (11) holds.

Proof of Proposition III.5. Given a type-2 corner point $(\alpha, \beta)$, we know from Proposition III.3 (i) that $\alpha=l_{1}^{\Omega_{F R}}(j)+$ 1 for some $j=1, \ldots, n_{2, \max }$. Choosing $n=l_{2}^{\Omega^{o}}(\alpha-1)-\beta \geq$ $0, m=\min \left\{\left(l_{2}^{\Omega_{F R}}(\alpha)-\beta\right), n\right\}, \hat{S}^{-}(n)=\left\{l_{1}^{\Omega_{F R}^{2}}\left(j^{(2, u)}+1\right)+\right.$ $\left.1, \ldots, l_{1}^{\Omega_{F R}}\left(j^{(2, u)}\right)\right\} \times\{\beta, \ldots, \beta+n\} \subseteq \Omega^{o}$, and $\hat{S}^{+}(m)=$ $\left\{l_{1}^{\Omega_{F R}}\left(j^{(2, l)}\right)+1, \ldots, l_{1}^{\Omega_{F R}}\left(j^{(2, l)}-1\right)\right\} \times\{\beta, \ldots, \beta+m\} \subseteq$ $\Omega_{F R} \backslash \Omega^{o}$ (see Figure 5), it follows that the sets $\Omega^{o} \backslash \hat{S}^{-}(n)$ 


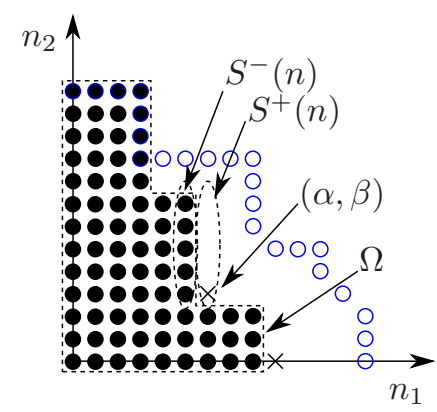

Figure 4. An example of a coordinate-convex set $\Omega$ having a type- 2 corner point $(\alpha, \beta)$ for which $\alpha \neq l_{1}^{\Omega_{F R}}(j)+1$ for every $j=1, \ldots, n_{2, \max }$.

and $\Omega^{o} \cup \hat{S}^{+}(m)$ are coordinate-convex, so $\hat{S}^{-}(n)$ is $I R_{\Omega^{\circ}}$ and $\hat{S}^{+}(m)$ is $I A_{\Omega^{\circ}}$. By (7) we get

$$
\begin{gathered}
J\left(\hat{S}^{-}(n)\right) \\
=r_{1} x_{1}\left(l_{1}^{\Omega_{F R}}\left(j^{(2, u)}+1\right)+1, l_{1}^{\Omega_{F R}}\left(j^{(2, u)}\right)\right)+r_{2} x_{2}(\beta, \beta+n)
\end{gathered}
$$

and

$$
\begin{gathered}
J\left(\hat{S}^{+}(m)\right) \\
=r_{1} x_{1}\left(l_{1}^{\Omega_{F R}}\left(j^{(2, l)}\right)+1, l_{1}^{\Omega_{F R}}\left(j^{(2, l)}-1\right)\right)+r_{2} x_{2}(\beta, \beta+m) .
\end{gathered}
$$

Combining these equalities with [1, Lemma 2] (which implies $\left.J\left(\hat{S}^{-}(n)\right) \geq J\left(\hat{S}^{+}(n)\right)\right)$, we have

$$
\begin{array}{ll} 
& R\left(x_{2}(\beta, \beta+n)-x_{2}(\beta, \beta+m)\right) \\
\geq \quad & x_{1}\left(l_{1}^{\Omega_{F R}}\left(j^{(2, l)}\right)+1, l_{1}^{\Omega_{F R}}\left(j^{(2, l)}-1\right)\right) \\
& -x_{1}\left(l_{1}^{\Omega_{F R}}\left(j^{(2, u)}+1\right)+1, l_{1}^{\Omega_{F R}}\left(j^{(2, u)}\right)\right) .
\end{array}
$$

Since $0 \leq n-m \leq B_{2}$ and $\lambda_{2}(\cdot)$ is nonincreasing, by [1, Lemma 3] we get

$$
x_{2}\left(0, B_{2}\right) \geq x_{2}(\beta, \beta+n)-x_{2}(\beta, \beta+m)
$$

which, when combined with (18), proves (14). Formula (ii) is obtained in the same way by exchanging the roles of the two classes of users.

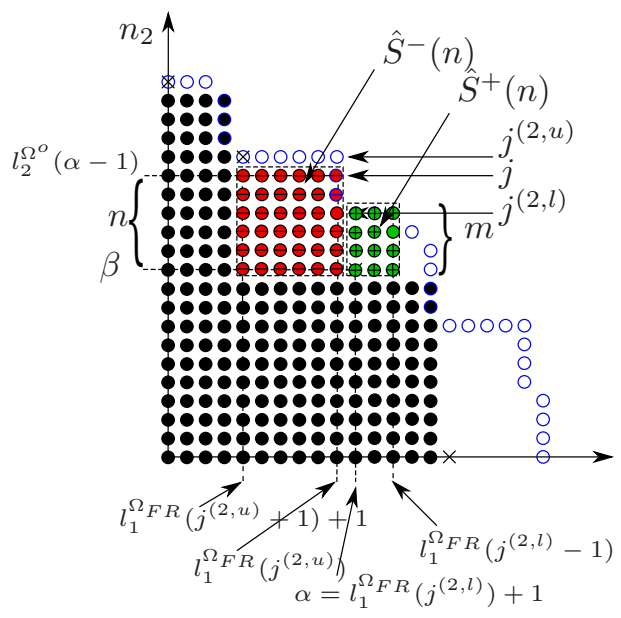

Figure 5. A description of a step in the proof of Proposition III.5.
Proof of Theorem III.6. (i) If $\frac{1}{R}<L_{1}$, then by Proposition III.5 (ii) $\Omega^{o}$ has no type- 1 corner points, so it is a threshold type- 1 policy by [1, Lemma 1$]$. Let $t_{1}$ denote the corresponding threshold. Then either $t_{1}=n_{1, \max }=l_{1}^{\Omega_{F R}}(0)$ or $\left(t_{1}+1,0\right)$ is a type- 2 corner point for $\Omega^{o}$. In the second case, by Proposition III.3 (i) we have $t_{1}+1=l_{1}^{\Omega_{F R}}(j)+1$ for some $j=1, \ldots, n_{2, \max }$.

(ii) is proved similarly.

(iii) If $\frac{1}{L_{1}}<R<L_{2}$, then by parts (i) and (ii) $\Omega^{o}$ is both threshold type- 1 and threshold type-2, so it coincides with $\Omega_{F R}$.

Proof of Corollary III.7. For each $j=0, \ldots, n_{1, \max }$, it follows from the definitions of $x_{2}(\cdot, \cdot)$ and of $j^{(1, l)}, j^{(1, u)}$ that

$$
\begin{gathered}
x_{2}\left(l_{2}^{\Omega_{F R}}\left(j^{(1, l)}\right)+1, l_{2}^{\Omega_{F R}}\left(j^{(1, l)}-1\right)\right) \geq l_{2}^{\Omega_{F R}}\left(j^{(1, l)}\right)+1, \\
x_{2}\left(l_{2}^{\Omega_{F R}}\left(j^{(1, u)}+1\right)+1, l_{2}^{\Omega_{F R}}\left(j^{(1, u)}\right)\right) \leq l_{2}^{\Omega_{F R}}\left(j^{(1, u)}\right),
\end{gathered}
$$

and $l_{2}^{\Omega_{F R}}\left(j^{(1, u)}\right)=l_{2}^{\Omega_{F R}}\left(j^{(1, l)}\right)$, so $L_{1} \geq \frac{1}{x_{1}\left(0, B_{1}\right)}$. Similarly, we have $L_{2} \geq \frac{1}{x_{2}\left(0, B_{2}\right)}$.

\section{REFERENCES}

[1] K. Ross and D. Tsang, "The stochastic knapsack problem," IEEE Transactions on Communications, vol. 37, no. 7, pp. 740-747, 1989.

[2] W. Golab and R. Boutaba, "Admission control in data transfers over lightpaths," IEEE Journal on Selected Areas of Communications, vol. 25, no. 6, pp. 102-110, 2007.

[3] H. El-Sayed, A. Mellouk, L. George, and S. Zeadally, "Quality of service models for heterogeneous networks: overview and challenges," Annals of Telecommunications, vol. 63, no. 11-12, pp. 639-668, 2008.

[4] K. W. Ross, Multiservice Loss Models for Broadband Telecommunication Networks. Springer, New York, 1995.

[5] A. Dasylva and R. Srikant, "Bounds on the performance of admission control and routing policies for general topology networks with multiple call classes," in Proc. IEEE INFOCOM, vol. 2, 1999, pp. 505-512.

[6] B. L. Miller, "A queueing reward system with several customer classes," Management Science, vol. 6, no. 13, pp. 234-245, 1969.

[7] E. Altman, T. Jiménez, and G. Koole, "On optimal call admission control in resource-sharing system," IEEE Transactions on Communications, vol. 49, no. 9, pp. 1659-1668, 2001.

[8] J. Ni, D. Tsang, S. Tatikonda, and B. Bensaou, "Threshold and reservation based call admission control policies for multiservice resourcesharing systems," in Proc. IEEE INFOCOM, vol. 2, 2005, pp. 773-783.

[9] _ - "Optimal and structured call admission control policies for resource-sharing systems," IEEE Transactions on Communications, vol. 55, pp. 158-170, 2007.

[10] M. Marchese, QoS Over Heterogeneous Networks. Wiley, 2007.

[11] S. Deng and U. Maydell, "Optimal control of flexible bandwidth calls in B-ISDN," in Proc. of ICC'93, vol. 3, 1993, pp. 1315-1319.

[12] T. Javidi and D. Teneketzis, "An approach to connection admission control in single-hop multiservice wireless networks with QoS requirements," IEEE Transactions on Vehicular Technology, vol. 52, pp. 1110$1124,2003$.

[13] N. Likhanov, R. R. Mazumdar, and F. Theberge, "Providing QoS in large networks: Statistical multiplexing and admission control," in Analysis, Control and Optimization of Complex Dynamic Systems, E. Boukas and R. Malhame, Eds. Kluwer, 2005.

[14] M. Cello, G. Gnecco, M. Marchese, and M. Sanguineti, "CAC with nonlinearly-constrained feasibility regions," IEEE Communications Letters, 2011, to appear. 